\title{
Efeitos do autocontrole da sequência de movimentos na aprendizagem de uma tarefa de "timing" coincidente
}

CDD. 20.ed. 152.3

http://dx.doi.org/10.1590/1807-55092014000400651

\author{
Flavio Henrique BASTOS* \\ Ulysses Okada de ARAUJO* \\ Andrea Michele FREUDENHEIM* \\ *Escola de Educa- \\ ção Física e Esporte, \\ Universidade de São \\ Paulo.
}

\section{Resumo}

0 objetivo do presente estudo foi investigar os efeitos de autocontrolar a sequência de movimentos, na aprendizagem de uma tarefa de "timing" coincidente. Trinta adultos jovens (idade $=23 \pm 5,5$ anos) sem experiência prévia na tarefa foram distribuídos aleatoriamente em dois grupos com condições diferentes quanto a sequência de movimentos, autocontrolado (LIVRE) e externamente controlado (DET). Os participantes deveriam tocar seis sensores sequencialmente, o último coincidentemente à chegada de um estímulo visual (tarefa de "timing"). Na aquisição, os participantes do grupo DET deveriam tocar os sensores numa sequência determinada, enquanto os do grupo LIVRE poderiam tocar os sensores em qualquer ordem (exceto o último, que foi o mesmo para ambos os grupos). Apesar de nenhuma diferença ter sido encontrada em relação ao número de tentativas necessárias ao alcance do desempenho critério na aquisição, o grupo LIVRE apresentou pior desempenho na transferência comparado ao grupo DET, indicando que ter controle sobre a sequência de movimentos que precede a coincidência prejudica a aprendizagem da habilidade.

Palavras-chave: Aprendizagem motora; Aprendizagem autocontrolada; Aprendizagem de sequências; Estratégias de aprendizagem.

\section{Introdução}

Nas últimas duas décadas, os efeitos de condições autocontroladas na aprendizagem de habilidades motoras tornou-se objeto de uma série de estudos, em razão dos resultados benéficos encontrados. Experimentos que investigam estas condiçôes concedem aos aprendizes o controle sobre algum aspecto da prática que seria controlado externamente por um experimentador. As evidências encontradas indicam que o processo de aprendizagem é beneficiado quando os aprendizes podem escolher quando visualizar um modelo ${ }^{1}$, quando receber "feedback" aumentado ${ }^{2,3-7}$, qual a dificuldade da tarefa que desejam praticar ${ }^{8}$, a quantidade de tentativas de prática $^{9}$ e a organizaçáo da prática ${ }^{2,10-12}$. O conjunto de evidências indica que os benefícios não estáo restritos a um único fator que afeta a aprendizagem (e.g. "feedback" aumentado), ou a um tipo de tarefa específico (e.g. tarefa seriada), mas que se trata de um efeito generalizável.

As principais hipóteses explicativas para os benefícios da prática autocontrolada foram adaptadas $\mathrm{da}$ aprendizagem cognitiva ${ }^{13}$. Duas principais linhas de interpretação buscam explicar os benefícios da prática autocontrolada. A primeira delas tem como foco processos cognitivos, como a elaboração de estratégias de aprendizagem para o alcance de um objetivo ou meta ${ }^{14}$. Estes processos levariam a um aumento do esforço cognitivo durante a prática em relação ao que se daria sem que houvesse controle por parte do aprendiz. Os ganhos de aprendizagem, observáveis nas fases de transferência e retenção, seriam obtidos por mecanismos similares aos apontados por LEE et al..$^{15}$ associados à prática variada, ao uso de modelos iniciantes e à baixa frequência de fornecimento de "feedback" aumentado. A segunda linha de interpretação sobre os ganhos de aprendizagem obtidos em condiçôes autocontroladas tem como foco processos motivacionais. De acordo com alguns autores ${ }^{16}$, a Teoria da Auto-Determinação (Self-determination Theory), um coletivo de teorias, serviria de base para interpretaçóes sobre os mecanismos subjacentes aos ganhos de aprendizagem 
observados. Dentre as principais consideradas pelos autores estariam a Teoria de Necessidades Psicológicas Básicas (Basic Psychological Needs Theory), a Teoria da Avaliação Cognitiva (Cognitive Evaluation Theory) e a Teoria da Integração Organísmica (Organismic Integration Theory) - veja SANLI et al. ${ }^{16}$, para uma revisão e compreensão das relaçôes entre as proposiçóes da Teoria da Auto-determinação e as ideias presentes em estudos sobre a prática autocontrolada.

Mais especificamente, alguns autores sugerem que a prática autocontrolada pode beneficiar a aprendizagem por incentivar os aprendizes a explorar mais estratégias de movimento em relação a uma condição em que isso não é possível ${ }^{2,17}$. No estudo de Wulf e Toole ${ }^{2}$, por exemplo, foram comparadas uma condição na qual os indivíduos podiam decidir quando usar bastôes ("poles"), que serviam como apoio em um simulador de esqui, e uma condição espelhada ("yoked"). Ambos os grupos alcançaram amplitude de movimento semelhante durante a fase de aquisição, mas o grupo na condição de prática autocontrolada apresentou melhor desempenho na retenção. Este ganho de aprendizagem, observado na retenção, foi explicado pela possibilidade do grupo autocontrolado testar estratégias de movimento durante a aquisição, por meio do uso dos bastóes.

Em BAstos et al. ${ }^{17}$, com o objetivo de investigar se o número de elementos fixos de uma sequência (disponível para autocontrole) afeta a prática de uma tarefa motora, foi utilizada uma tarefa que permitia que os aprendizes decidissem, a cada tentativa, a própria sequência de movimentos. No referido estudo, os aprendizes deveriam executar uma sequência de seis movimentos com a mão dominante, tocando seis sensores, de forma a viabilizar a interação adequada com a chegada de um estímulo externo ("timing" coincidente). O início do deslocamento do estímulo foi o sinal para que o executante iniciasse a sequência de movimentos. Assim, a janela temporal para interagir com o estímulo, ao final da sequência de movimentos, seria maior ou menor em função do tempo demandado pela sequência. Por exemplo, uma determinada sequência de movimentos poderia demandar maior deslocamento da mão no espaço, ou conter um maior número de reversōes, demandando mais tempo para a sua execução e fazendo com que o executante não tivesse, ao final da sequência, condiçóes de interagir adequadamente com o estímulo, levando a uma resposta de "timing" atrasada. Os autores encontraram evidência de que, podendo escolher a própria sequência de movimentos na tarefa descrita, os aprendizes buscam por aquelas que apresentam menor quantidade de reversóes. Este tipo de sequência, de execução mais confortável e fluida ${ }^{18}$, possibilita chegar ao último elemento $\mathrm{da}$ sequência com uma maior disponibilidade de tempo (janela temporal) para responder adequadamente à chegada do estímulo externo.

Como frisado, o estudo de BAstos et al. ${ }^{17}$ verificou os efeitos de autocontrolar a sequência de movimentos que antecedem a coincidência, na prática de uma tarefa de "timing" coincidente. Estes resultados corroboram os de Wulf e Toole 2 , pois foi observado que os aprendizes em condição de prática autocontrolada testam estratégias a partir das soluçôes motoras disponíveis (sequências de movimentos) na fase de aquisição. Assim, se em uma tarefa de "timing" coincidente os aprendizes testam estratégias de movimento quando estão em uma condição de prática autocontrolada ${ }^{17}$, há a possibilidade de que autocontrolar a sequência de movimentos afete sua aprendizagem. Portanto, o objetivo do presente estudo foi investigar os efeitos de autocontrolar a sequencia de movimentos, na aprendizagem de uma tarefa de "timing" coincidente. Mais especificamente, investigar os efeitos de oferecer o controle da sequência de movimentos aos aprendizes, na aprendizagem de uma tarefa de "timing" coincidente que depende desta sequência.

A tarefa utilizada no presente estudo foi a mesma de Bastos et al. ${ }^{17}$. Um exemplo de habilidade motora simulada por esta tarefa experimental, na área de Educação Física e Esporte, seria a recepção do voleibol. Especificamente, nesta tarefa há necessidade do jogador que irá receber a bola realizar sequências de movimento para ajustar a posição de seu corpo e, ao término destes ajustes, interagir com a bola de maneira adequada ("timing" antecipatório).

Se permitir aos aprendizes explorar sequências de movimento em uma tarefa com demanda de tempo pode beneficiar a aprendizagem, poder-se-ia esperar diferenças entre os grupos quanto à quantidade de prática na aquisição e também um melhor desempenho na transferência. No entanto, Bund e WieMEYER ${ }^{12}$, consideram que há uma relação entre estes processos cognitivos e motivacionais envolvidos na aprendizagem motora, numa condição de prática autocontrolada. De acordo com os autores, controlar a prática aumenta a motivação - o que levaria a um ganho de desempenho durante a aquisição de uma habilidade motora - e ser responsável por este controle aumenta também a demanda de processamento cognitivo, podendo levar a um desempenho inferior na aquisição, em comparação a uma condição na qual o aprendiz 
não precisa controlar sua prática (prática externamente controlada). Desta forma, o ganho de aprendizagem dos aprendizes controlando a prática seria observado somente em testes de transferência e retenção, mas não durante o processo de aquisição da habilidade.

Portanto, se autocontrolar a sequência de movimentos envolve maior demanda cognitiva, de

\section{Método}

\section{Amostra}

Participaram do experimento 30 voluntários (grupo DET: média de idade $=26,2$ anos, DP $=5,7$; grupo LIVRE: média de idade $=23,6, \mathrm{DP}=5,5)$, de ambos os sexos (grupo DET: nove participantes do sexo masculino e seis do feminino; grupo LIVRE: seis participantes do sexo masculino e nove do feminino), sem experiência prévia na tarefa. $\mathrm{O}$ estudo foi aprovado pelo Comitê de Ética local e todos os participantes assinaram um termo de consentimento (protocolo n o 2006/08).

\section{Instrumentos e tarefas}

O aparelho consistiu em uma canaleta de $200 \mathrm{~cm}$ com 96 LEDs (diodos emissores de luz) - o primeiro e o último LED sendo o sinal de atenção e o LED alvo, acordo com o antagonismo entre os processos motivacionais e cognitivos proposto por Bund e WIEMEYER $^{12}$, possíveis efeitos benéficos da prática autocontrolada nessa tarefa experimental não seriam observados, quanto à quantidade de prática para atingir o critério, na fase de aquisição, mas somente em relação ao desempenho na fase de transferência. respectivamente (FIGURA 1). Estes LEDs acendem sequencialmente, de modo a criar um movimento aparente da luz através da canaleta a uma velocidade constante de $1,33 \mathrm{~m} / \mathrm{s}$.

A canaleta formava um ângulo de aproximadamente $10^{\circ}$ em relação a seu suporte horizontal, e foi posicionada a $10 \mathrm{~cm}$ de uma mesa de madeira $(70$ $\times 90 \times 6 \mathrm{~cm}$ ), com aberturas, nas quais seis sensores sensíveis ao toque $(5 \times 15 \mathrm{~cm}$ cada) foram encaixados e um visor de "feedback" (24 x $8 \mathrm{~cm}$ ) com cinco LEDs alinhados correspondendo as cinco mensagens de "feedback" possíveis.

Essas mensagens de "feedback" foram ajustadas da seguinte forma: a) "muito antes", fornecida quando o último toque foi feito mais de $60 \mathrm{~ms}$ antes da ativação do último LED; b) "antes", mais de $30 \mathrm{~ms}$ antes; c) "correto", dentro de $\pm 30 \mathrm{~ms}$; d) "depois", mais de 30 ms depois; e, e) "muito depois", mais de 60 ms depois.

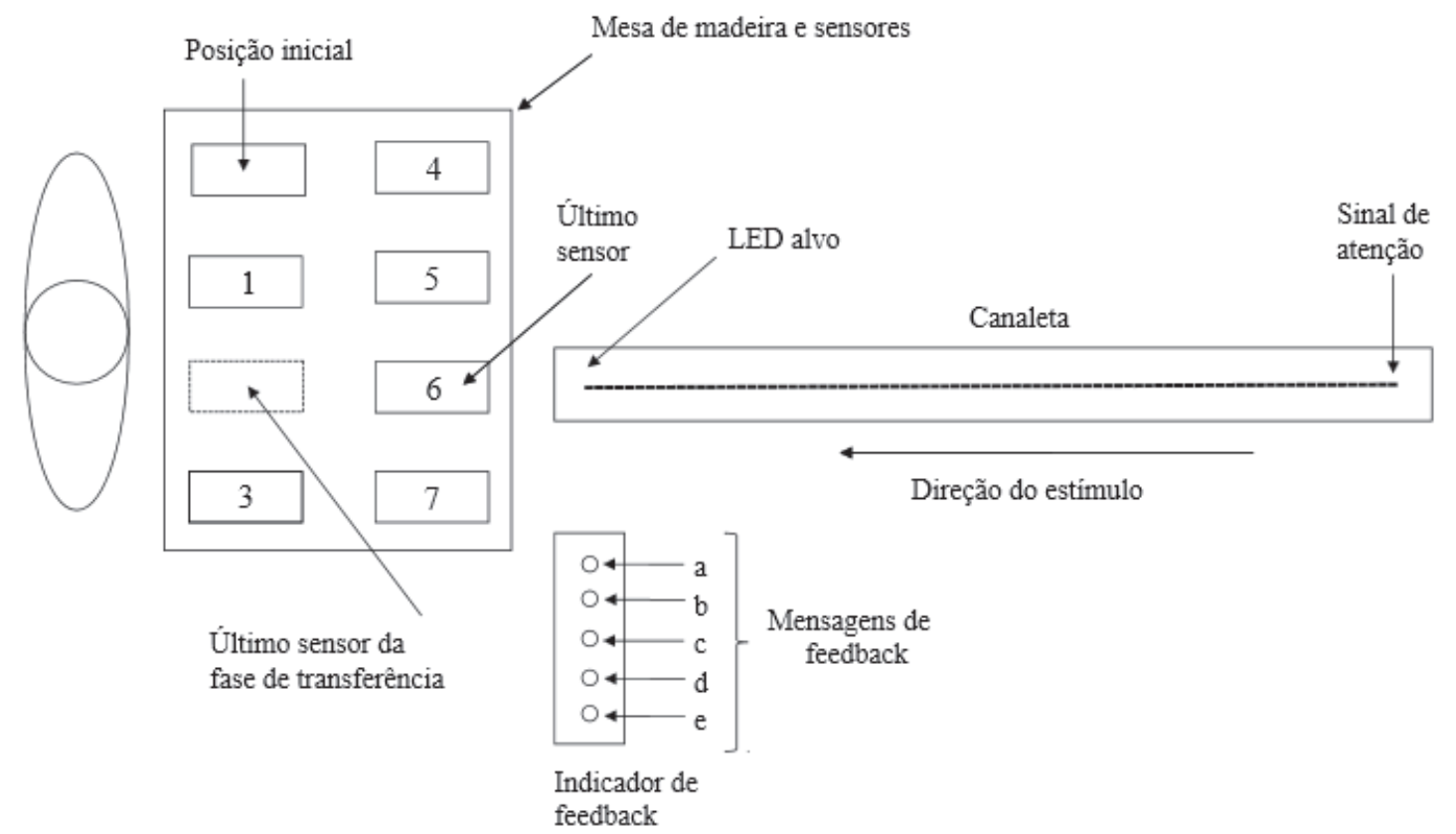

Os números mencionados não estavam presentes durante 0 experimento. Um cartão indicando os sensores que tinham de ser tocados (e a ordem na qual eles tinham que ser tocados pelo grupo DET) cobria o último sensor da fase de transferência durante a fase de aquisição e cobria 0 último sensor da fase de aquisição durante a fase de transferência. As letras de 'a' a ' $e$ ' indicam quais LEDs estavam associados a quais "feedbacks".

FIGURA 1 - Esquema do equipamento com os sensores numerados para uma melhor compreensão das sequências de movimentos mencionadas no texto. 
O aparelho foi conectado a um computador equipado com "software" desenvolvido especificamente para o estudo, que controlava a ativação dos LEDs, registrava a sequência em que os sensores foram tocados e a diferença de tempo (milissegundos) entre o último toque e o acendimento do LED alvo.

A velocidade do estímulo visual foi ajustada em $1,33 \mathrm{~m} / \mathrm{s}$ na fase de aquisição e em $1,17 \mathrm{~m} / \mathrm{s}$ na de transferência com base em estudos anteriores usando o mesmo aparelho e em um estudo piloto. $\mathrm{O}$ final da canaleta (LED alvo) foi posicionado próximo do último sensor a ser tocado, de modo que o estímulo - movimento aparente da luz - correu em direção aos participantes num plano sagital. A posição do último sensor a ser tocado foi escolhida de modo a evitar que a sua posição pudesse induzir a existência de apenas uma sequência de movimento para a realização da tarefa.

A tarefa experimental consistiu em tocar os seis sensores sequencialmente, de forma que o sexto e último toque coincidisse com a chegada do estímulo luminoso, ou seja, com o acendimento do LED alvo.

\section{Delineamento experimental e procedimentos}

O experimento consistiu de duas fases experimentais: aquisição e transferência. Os participantes foram designados aleatoriamente para um dos dois grupos experimentais, chamados LIVRE e DET. Para os participantes do grupo DET, a ordem da sequência foi definida previamente. Os participantes deste grupo tiveram que tocar os sensores 1, 3, 7, 5, 4 e o último, nesta ordem. Esta sequência foi determinada a partir da sequência mais utilizada por participantes de um estudo-piloto que praticaram a tarefa em condiçóes idênticas ao grupo LIVRE. Por sua vez, os participantes do grupo LIVRE não tiveram a sequência definida, somente o último sensor (LED alvo), que foi o mesmo para ambos os grupos. Um esquema da sequência na qual os sensores tinham que ser tocados estava disponível durante a fase de aquisição para ambos os grupos, e incluía a ordem de todos os toques (grupo DET) ou os sensores de posição inicial e coincidência (grupo LIVRE). Este cartão cobria o sensor que foi utilizado somente na fase de transferência para evitar confusão pelos participantes. $\mathrm{Na}$ fase de transferência um cartáo semelhante contendo a ordem de todos os toques foi apresentado para ambos os grupos, e cobria o sensor utilizado na aquisiçáo que não deveria ser tocado na fase de transferência.
A coleta foi individual, somente com um experimentador presente, em sala especialmente preparada para esta finalidade. Todos os participantes foram instruídos sobre o estímulo visual que iria "se mover" em sua direçáo assim que o LED de atenção apagasse, o sensor a ser tocado coincidentemente com o acendimento do LED alvo e que deveriam manter a mão direita tocando o sensor denominado "posição inicial" (FIGURA 1) até que o LED de atenção apagasse. Este procedimento foi empregado com a intençáo de dar a todos o mesmo tempo para tocar os sensores. $\mathrm{O}$ deslocamento do estímulo visual não era iniciado se o participante não estivesse tocando o sensor de posição inicial.

O grupo DET foi instruído sobre a ordem em que os sensores tinham que ser tocados, enquanto o grupo LIVRE foi informado sobre os sensores que tinham de tocar, em qualquer ordem, antes de tocar o último, e que a eles era permitido alterar a sequência utilizada a qualquer momento. Ambos os grupos também foram informados de que receberiam "feedback" após cada tentativa e que deveriam tentar receber o máximo possível do "feedback" "correto".

A fase de aquisiçāo terminava quando os participantes chegavam a um desempenho critério de três tentativas consecutivas com o último toque sendo feito no intervalo de tempo "correto" ( $\pm 30 \mathrm{~ms}$ ). Esta faixa de erro foi determinada a partir de estudos com "timing" coincidente em que os participantes mantiveram o erro abaixo deste patamar ${ }^{19}$ e estudo-piloto. Imediatamente após o final da fase de aquisiçáo os participantes foram instruídos sobre a tarefa da fase de transferência. Esta fase experimental foi idêntica para ambos os grupos, com a apresentação de uma nova sequência de toques com ordem definida (sensores 1, 4, 3, 5, 7 e um novo sensor - anteriormente oculto - designado para ser o último da fase de transferência - ver FIGURA 1) e uma modificaçáo da velocidade do estímulo visual $(1,33 \mathrm{~m} / \mathrm{s}$ para 1,17 $\mathrm{m} / \mathrm{s})$. Na fase de transferência todos os participantes realizaram 30 tentativas sem conhecimento de resultados.

\section{Análise dos dados}

As medidas foram calculadas para blocos de seis tentativas. Como o número de tentativas necessário para terminar a fase de aquisição diferiu entre os participantes (desempenho critério), o desempenho nessa fase foi avaliado considerando-se as seis primeiras e as seis últimas tentativas realizadas pelos participantes. Todas as tentativas realizadas na fase de transferência foram consideradas (cinco blocos de seis tentativas).

Os erros absoluto (EA) e constante (EC) foram calculados para indicar a magnitude e a direção do 
erro, respectivamente. As medidas foram calculadas utilizando a diferença de tempo entre o acendimento do LED alvo (FIGURA 1) e o toque no último sensor. Assim, o erro absoluto é a média do desempenho obtido, em um dado bloco de seis tentativas, desprezando a antecipação ou o atraso do erro, enquanto o erro constante é a média do mesmo desempenho obtido considerando a direção. O erro variável (EV), que reflete a consistência do desempenho, também foi calculado com base na mesma diferença de tempo, a partir da raiz quadrada do somatório do quadrado da diferença entre o desempenho de cada tentativa no bloco e a média do desempenho no mesmo bloco.

Foi considerado para análise, além disso, o número de vezes que os participantes do grupo LIVRE mudaram a sequência utilizada. Mais especificamente, avaliou-se o número de mudanças durante toda a fase de aquisição e depois se discriminou quantas delas foram realizadas na primeira e na última metade das tentativas da fase de aquisição.

Os pressupostos de normalidade (teste ShapiroWilk) e homogeneidade de variância (teste de

\section{Resultados}

\section{Fase de aquisição}

\section{Número de tentativas}

Não foi detectada diferença entre grupos no que se refere ao número de tentativas na fase de aquisição, $\mathrm{F}(1,28)=0,80 ; \mathrm{p}=0,77$. Isso exclui possíveis efeitos da condição de prática nessa variável, assim como hipóteses explicativas para os resultados relacionadas a quantidades de prática diferentes entre os grupos.

\section{Erro absoluto}

Ambos os grupos diminuíram o erro absoluto do primeiro para o último bloco da aquisição, como é possível observar no gráfico superior da FIGURA 2. Houve efeito principal para o fator Bloco, $\mathrm{F}(1,28)=$ 23,46; $\mathrm{p}<0,001$. Não foi detectado efeito para o fator Grupo $F(1,28)=1,42 ; p=0,242$ ou interação Grupo $x$ Bloco $F(1,28)=1,84 ; p=0,185$. Este resultado era esperado pois os participantes foram aleatoriamente distribuídos entre grupos, minimizando possíveis diferenças entre participantes, e todos os participantes atingiram um critério de desempenho - de forma que as últimas três tentativas de todos os participantes tiveram erro abaixo de $30 \mathrm{~ms}$.
Levene) foram verificados. A fim de satisfazer esses pressupostos, valores extremos (acima de dois desvios-padrão) foram removidos - não mais do que dois valores para cada participante no mesmo bloco de tentativas. Como resultado deste procedimento, $6 \%$ dos dados originais foram removidos da análise.

Para as medidas de erro na fase de aquisição e para o número de tentativas de prática nesta fase foram realizadas análises de variância (ANOVAs) de dois fatores (Grupo x Bloco), com medidas repetidas no segundo fator. $\mathrm{O}$ mesmo teste foi aplicado para as medidas de erro na fase de transferência. Quando necessário, foi empregada a correção Greenhouse-Geisser para esfericidade, além de testes t pareados com o ajuste de comparaçóes múltiplas de Benjamini-Hochberg ${ }^{20}$ como teste "post-hoc" para localizar diferenças entre blocos. $\mathrm{O}$ número de tentativas da fase de aquisição foi testado utilizandose uma ANOVA de um fator (grupos). O nível de significância adotado para os testes foi de $\alpha<0,05$.

A organização dos dados, bem como as análises e gráficos foram realizados utilizando o "software" $\mathrm{R}$ 21.

\section{Erro constante}

Os resultados do erro constante para ambos os grupos na fase de aquisição podem ser observados no gráfico central da FIGURA 2. Foi detectada diferença para o efeito principal no fator Bloco $\mathrm{F}(1$, 28) $=19,49 ; \mathrm{p}<0,001$, mas não no efeito principal no fator Grupo ou na interação Grupo x Bloco, F(1, 28) $=1,34 ; \mathrm{p}=0,257$ e $\mathrm{F}(1,28)=1,62 ; \mathrm{p}=0,213$ respectivamente. De forma similar ao erro absoluto, ambos os grupos diminuíram o erro constante do primeiro para o último bloco da aquisição.

\section{Erro variável}

Os resultados para o erro variável na fase de aquisição, ilustrados no painel inferior da FIGURA 2, são semelhantes aos apresentados nos erros absoluto e constante. A redução do erro variável do primeiro para o último bloco da aquisição foi apontada no efeito principal de Bloco, $\mathrm{F}(1,28)=16,89$; $\mathrm{p}<$ 0,001 . Não foi detectada interação entre Grupo e Bloco $\mathrm{F}(1,28)=0,04 ; \mathrm{p}=0,840$ ou efeito para o fator Grupo $\mathrm{F}(1,28)=0,24 ; \mathrm{p}=0,625$. 


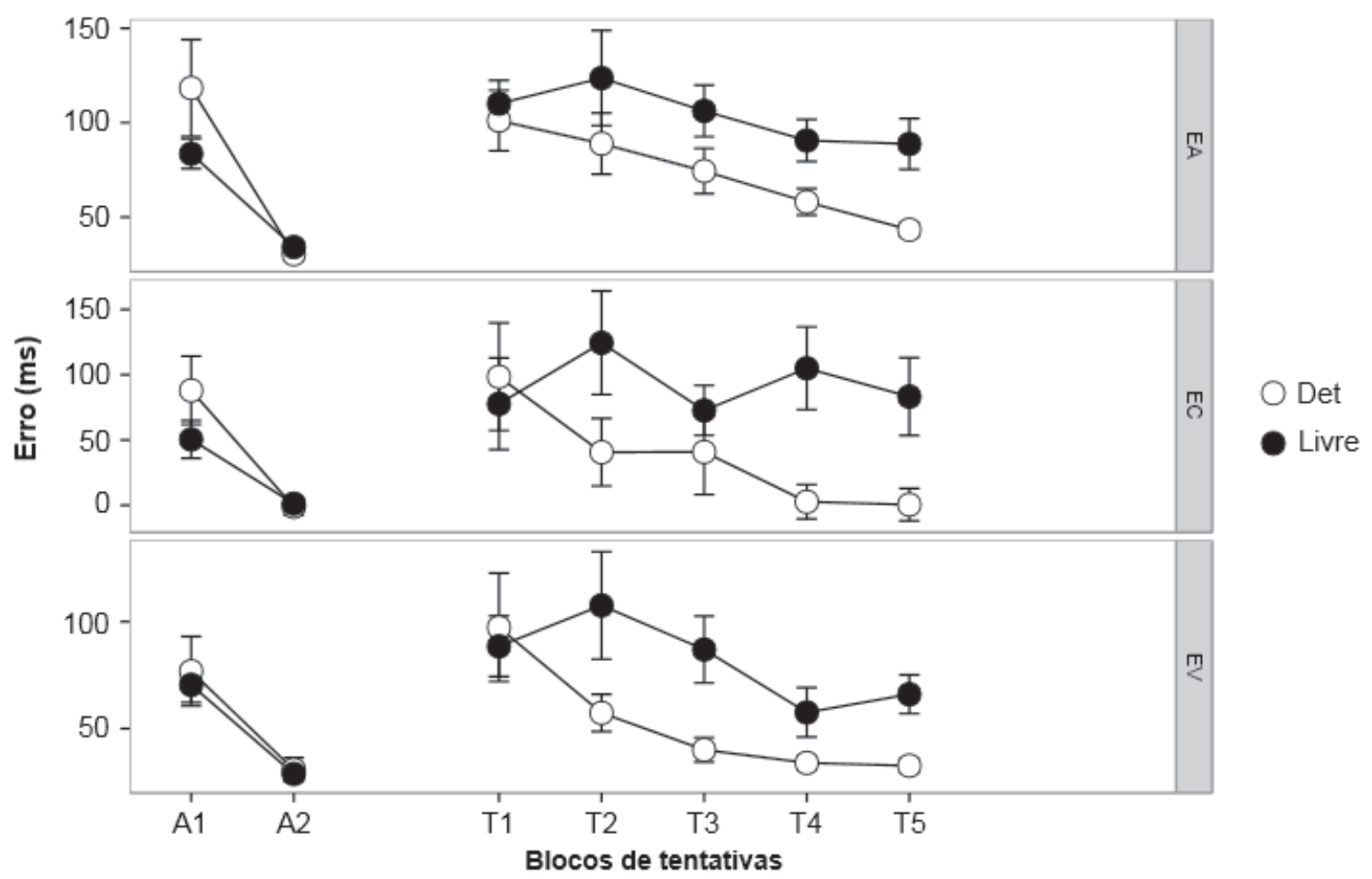

FIGURA 2 - Médias dos Erros absoluto, constante e variável dos grupos DET e LIVRE nas primeiras e últimas seis tentativas da fase de aquisição (A1 e A2) e durante a fase de transferência (T1-T5).

Mudança de sequência

Dos 15 participantes do grupo LIVRE, seis utilizaram a mesma sequência durante toda a fase de aquisiçáo. Sete participantes utilizaram mais de uma sequência na primeira metade das tentativas, mas apenas dois destes modificaram a sequência realizada na segunda metade das tentativas. Isto sugere possíveis estratégias empregadas pelos participantes no sentido de explorar diferentes sequências no início da prática e reduzir a exploração ao longo do tempo, provavelmente em busca de melhor desempenho.

\section{Fase de transferência}

\section{Erro absoluto}

O erro absoluto para os grupos LIVRE e DET durante a transferência pode ser observado no gráfico superior da FIGURA 2. Ambos os grupos reduziram o erro durante a transferência de forma detectável no efeito principal do fator Bloco $\mathrm{F}(4,112)=4,58$; $p=0,002$, com as diferenças localizadas entre $o$ primeiro e o segundo blocos comparados ao quarto bloco ( $p=0,026$ e $p=0,043$ respectivamente) e entre os três primeiros blocos e o quinto bloco $(\mathrm{p}=$ 0,026 para as três comparaçóes). Todavia, o grupo
DET manteve o erro absoluto em nível inferior ao grupo LIVRE durante toda a transferência, como reflete a diferença encontrada no fator Grupo, F(1, 28) $=5,53 ; p=0,026$. Náo foi detectada interação entre Grupo e Bloco F(4, 112) = 0,62; p = 0,598.

\section{Erro constante}

O gráfico central da FIGURA 2 ilustra os resultados para o erro constante na fase de transferência. Embora tenha havido pequena superioridade do grupo DET e reduçáo do erro ao longo dos blocos para este grupo, estas tendências não foram confirmadas pelo teste, que não apontou interação Grupo $x$ Bloco $F(4,112)=2,82 ; \mathrm{p}=0,051$ ou efeito para os fatores Grupo F $(1,28)=2,98 ; \mathrm{p}=0,095$ e Bloco $\mathrm{F}(4,112)=1,75 ; \mathrm{p}=0,175$.

Erro variável

Os resultados para o erro variável na fase de transferência, ilustrados no painel inferior da FIGURA 2, se assemelham aos resultados para o erro absoluto. Apesar da redução do erro variável de ambos os grupos ao longo da transferência, confirmada na diferença no fator Bloco $\mathrm{F}(4,112)=$ 4,$89 ; \mathrm{p}=0,005$, o grupo DET manteve menor erro 
variável comparado ao grupo LIVRE durante toda a transferência, verificada no efeito principal para o fator Grupo $F(1,28)=6,15 ; p=0,002$. Portanto, embora ambos os grupos tenham se tornado menos variáveis ao longo da transferência, o grupo DET foi mais consistente. O "post-hoc" para o fator Bloco localizou as diferenças entre os dois primeiros

\section{Discussão}

Os benefícios de conceder aos aprendizes controle sobre sua prática têm se mostrado recorrente nos estudos de aprendizagem motora ${ }^{7}$. Nesse contexto, o objetivo do presente estudo foi investigar se oferecer o controle sobre a sequência de movimento que precede a coincidência favoreceria a aprendizagem de uma tarefa de "timing" coincidente. Este objetivo decorre da literatura apresentar que permitir aos aprendizes explorar sequências / estratégias de movimento como uma hipótese explicativa para o efeito benéfico da prática autocontrolada ${ }^{2}$. Desta forma, oferecer controle sobre a sequência de movimentos aos participantes do grupo LIVRE deveria proporcionar ganhos de aprendizagem, inferidos por meio de um melhor desempenho na fase de transferência, em relaçáo ao grupo em que este controle náo foi permitido (DET). $\mathrm{Na}$ fase de aquisiçáo, entretanto, não deveria ser observada nenhuma diferença entre os grupos, em função do antagonismo entre os processos motivacionais e cognitivos, proposto por BUnd e WiEMEYeR ${ }^{12}$.

Apesar da esperada ausência de diferenças entre os grupos quanto ao número de tentativas necessárias para atingir o critério de desempenho na aquisição, o grupo LIVRE apresentou maior EA e EC na fase de transferência em relação ao grupo DET, um resultado contrário à hipótese formulada. Este resultado opóe-se à hipótese de que o ganho de aprendizagem dos aprendizes controlando a prática poderia ser observado nos testes de transferência e retenção, mesmo que não o fosse durante o processo de aquisição, ou seja, a hipótese referente ao antagonismo entre motivação e esforço cognitivo ${ }^{12}$. Ainda, especificamente, ao contrário do que foi proposto por Wulf e Toole ${ }^{2}$, possibilitar a exploração de estratégias de movimento mostrou-se uma condição prejudicial à aprendizagem da habilidade motora.

Os resultados obtidos no presente estudo opóem-se a uma série de evidências encontradas na literatura que versa sobre prática autocontrolada em aprendizagem motora. Especificamente, quando comparadas a blocos e o quarto bloco $(\mathrm{p}=0,031$ para ambas as comparaçóes) e entre os dois primeiros blocos e o quinto bloco $(\mathrm{p}=0,031$ para a comparaçáo entre o primeiro e o quinto bloco e $\mathrm{p}=0,049$ para a comparação entre o segundo e o quinto bloco). Não foi detectada interação entre Grupo e Bloco, $\mathrm{F}(4$, 112) $=1,63 ; \mathrm{p}=0,195$.

condiçóes de prática controladas externamente, evidências têm indicado que condiçóes controladas pelos aprendizes proporcionam efeitos similares sobre a aprendizagem motora ${ }^{22-24} \mathrm{ou}$, criticamente, ganhos de aprendizagem ${ }^{2-12}$. Considerando a diversidade de tarefas experimentais e de fatores que afetam a aprendizagem manipulados nos estudos citados acima, faz-se essencial buscar explicaçóes para os resultados encontrados no presente estudo.

Um dos fatores sugeridos para os efeitos benéficos obtidos em condições de prática autocontrolada consiste destas condiçóes, em razão de suas características, aumentarem a motivação do aprendiz ${ }^{16}$. A Teoria das Necessidades Psicológicas Básicas, por exemplo, permitiria inferir que a autonomia proporcionada ao grupo LIVRE, em razáo da possibilidade de escolha que caracteriza a condição de prática deste grupo, deveria aumentar a motivaçáo dos participantes e, como consequência, levar a ganhos de aprendizagem. A condição de prática, por si, proporcionaria ganhos de aprendizagem. Entretanto, os resultados indicam que numa condição de prática, na qual a sequência de movimentos que precede a coincidência com um estímulo visual pode ser escolhida, há prejuízo para a aprendizagem da habilidade motora. Futuros estudos podem controlar o nível de motivação quando este tipo de controle é permitido ao aprendiz, tendo em vista que aspectos motivacionais tem-se mostrado um fator relevante, especialmente em estudos nos quais os aprendizes podem controlar a requisição de "feedback" aumentado (e.g. Chiviacowsky et al. ${ }^{25}$ ).

No estudo de Bastos et al. ${ }^{17}$ é possível observar que os aprendizes, numa condição semelhante à utilizada para o grupo LIVRE no presente estudo, apresentam diferentes padrōes de exploração das possibilidades de movimento. Estes diferentes padrōes sugerem que o efeito benéfico da prática autocontrolada pode dever-se não à condição em si, ou à possibilidade de exploração, mas ao uso que 
o aprendiz faz desta possibilidade. BAstos et al. ${ }^{14}$ obtiveram evidência de que uma meta de aprendizagem, uma referência oferecida aos aprendizes para o uso dos recursos numa condição autocontrolada, promove não somente diferentes estratégias de aprendizagem, como também diferentes efeitos na aprendizagem a partir de uma mesma condição de prática autocontrolada. Especificamente, da perspectiva do aprendiz, dois tipos diferentes de meta podem ser concebidos: a 'meta da tarefa', que se refere a realizar com sucesso a tarefa motora, e a 'meta de aprendizagem', que adiciona o contexto no qual a habilidade motora deverá ser realizada, também com sucesso. No caso do estudo de Bastos et al. ${ }^{14}$, a meta de aprendizagem utilizada consistiu em informar os participantes sobre como seria o teste que realizariam após um período de prática, de forma que pudessem utilizar a possibilidade de escolha, proporcionada pela condição autocontrolada, para preparar-se para o referido teste. Ambas as metas descritas não são mutuamente exclusivas, considerando que um aprendiz que possui uma meta de aprendizagem buscará a cada tentativa alcançar a meta da tarefa. Entretanto, no referido estudo o controle dos aprendizes esteve relacionado à escolha da ordem em que diferentes velocidades de um estímulo visual seriam praticadas, e não às possibilidades de movimento. Neste sentido, futuros estudos poderiam investigar se diferentes padrōes de utilização das possibilidades de exploração de movimentos, numa condição autocontrolada, proporcionam efeitos distintos sobre a aprendizagem de habilidades motoras. Adicionalmente, outra questão a ser investigada seria o efeito de oferecer uma meta de aprendizagem referente à exploração de possibilidades de movimento.

As mudanças de sequência feitas pelo grupo LIVRE na aquisição sugerem uma possível estratégia de explorar diferentes sequências no início da prática e reduzir a exploração mais tarde. Na tarefa proposta, os participantes do grupo LIVRE tinham dois objetivos: tocar (a) todos os sensores e (b) tocar o último sensor no momento certo. Considerando que o posterior depende do anterior, uma sequência melhor poderia permitir que os participantes melhorassem seu desempenho na tarefa de "timing". Assim, os aprendizes parecem ter se envolvido em um processo de busca no início da prática, visando descobrir uma sequência de movimento ótima e que, em seguida, tenham parado de testar sequências diferentes, a fim de melhorar o desempenho na coincidência utilizando a sequência escolhida. Esta "fading schedule" organização com maior variação no início e posterior reduçáo da variação - tem sido vista como positiva por combinar os benefícios de guiar os aprendizes a uma ideia geral do padrão de movimento correto já no início da prática e torná-los gradualmente independentes após um período de prática ${ }^{17-19}$. Entretanto, no que se refere especificamente à sequência autocontrolada de movimentos, esta forma de organização da prática não possibilitou melhor aprendizagem para o grupo LIVRE, quando comparado ao grupo DET, que teve sua sequência de movimento fixada desde o início da prática.

Em suma, os resultados do presente estudo permitem concluir que uma condiçáo de prática que permite aos aprendizes controlar a sequência de movimentos que antecede uma demanda de "timing" coincidente, prejudica a aprendizagem da habilidade. Futuros estudos podem investigar se fornecer este tipo de controle afeta a motivação dos aprendizes e se o mesmo efeito sobre a aprendizagem seria observado se considerados os padróes de exploração dos aprendizes, com e sem a presença de uma meta de aprendizagem.

\section{Abstract}

Effects of self-controlling the movement sequence on learning a coincident timing task

The purpose of this study was to investigate the effects of self-controlling a movement sequence on learning a coincident timing task. Thirty young adults (age $=23 \pm 5.5$ years) with no previous experience with the task were randomly assigned into two groups with different movement sequence conditions, self-controlled (FREE) and externally controlled (DET). Participants were required to tap six sensors sequentially, the last one coinciding with the arrival of a visual stimulus (timing task). On acquisition, participants in the DET group had to tap the sensors in a determined sequence, while those in the FREE group were allowed to tap the sensors in any order (except for the last one, which was the same for both groups). While no difference was found regarding the number of trials needed to reach the performance 
criterion in acquisition, the group FREE showed higher timing errors in transfer compared to the group $D E T$, indicating that self-controlling the movement sequence that precedes the coincidence impairs the learning of the motor skill.

KEY WORDS: Motor learning; Self-controlled learning; Sequence learning; Learning strategies.

\section{Referências}

1. Wulf G, Raupach M, Pfeiffer F. Self-controlled observational practice enhances learning. Res Q Exerc Sport. 2005;76:107-11.

2. Wulf G, Toole T. Physical assistance devices in complex motor skill learning: benefits of a self-controlled practice schedule. Res Q Exerc Sport. 1999;70:265-72.

3. Janelle CM, Kim J, Singer RN. Subject-controlled performance feedback and learning of a closed motor skill. Percept Mot Skills. 1995;81:627-34.

4. Janelle CM, Barba DA, Frehlich SG, Tennant LK, Cauraugh JH. Maximizing performance feedback effectiveness through videotape replay and a self-controlled learning environment. Res Q Exerc Sport. 1997;68:269-79.

5. Chiviacowsky S, Wulf G. Self-controlled feedback: does it enhance learning because performers get feedback when they need it? Res Q Exerc Sport. 2002;73:408-15.

6. Chiviacowsky S, Wulf G. Self-controlled feedback is effective if it is based on the learner's performance. Res Q Exerc Sport. 2005;76:42-8.

7. Chiviacowsky S, Godinho M, Tani G. Self-controlled knowledge of results: effects of different schedules and task complexity. J Hum Mov Stud. 2005;49:277-96.

8. Andrieux M, Danna J, Thon B. Self-control of task difficulty during training enhances motor learning of a complex coincidence-anticipation task. Res Q Exerc Sport. 2012;83:27-35.

9. Post PG, Fairbrother JT, Barros JAC. Self-controlled amount of practice benefits learning of a motor skill. Res Q Exerc Sport. 2011;82:474-81.

10. Walter C, Bastos FH, Araujo UO, Silva JAO, Corrêa UC. Estrutura de prática e liberdade de escolha na aprendizagem de habilidades motoras. Rev Port Cien Desp. 2008;8:337-46.

11. Keetch KM, Lee TD. The effect of self-regulated and experimenter-imposed practice schedules on motor learning for tasks of varying difficulty. Res Q Exerc Sport. 2007;78:476-86.

12. Bund A, Wiemeyer J. Self-controlled learning of a complex motor skill: effects of the learners' preferences on performance and self-efficacy. J Hum Mov Stud. 2004;47:215-36.

13. Wulf G. Self-controlled practice enhances motor learning: implications for physiotherapy. Physiotherapy. 2007;93:96-101.

14. Bastos FH, Marinovic W, de Rugy A, Tani G. Prior knowledge of final testing improves sensorimotor learning through self-scheduled practice. Hum Mov Sci. 2013;32:192-202.

15. Lee TD, Swinnen SP, Serrien DJ. Cognitive effort and motor learning. Quest. 1994;46:328-44.

16. Sanli EA, Patterson JT, Bray SR, Lee TD. Understanding self-controlled motor learning protocols through the selfdetermination theory. Front Psychol. 2013;3:611.

17. Bastos FH, Tani G, Araujo UO, Walter C, Freudenheim AM. Number of options in a movement sequence affects learners' behavior in a self-controlled practice condition. Percept Mot Skills. 2010;111:343-54.

18. Flash T, Hogan N. The coordination of arm movements: an experimentally confirmed mathematical model. J Neurosci. 1985;5:1688-703.

19. Williams LR, Jasiewicz JM, Simmons RW. Coincidence timing of finger, arm, and whole body movements. Percept Mot Skills. 2001;92:535-47.

20. Benjamini Y, Hochberg Y. Controlling the false discovery rate: a practical and powerful approach to multiple testing. J R Stat Soc Ser B Methodol. 1995;57:289-300.

21. R Development Core Team. R: a language and environment for statistical computing. Vienna: R Foundation for Statistical Computing; 2013. Available from: http://www.R-project.org/.

22. Boyce BA. Effects of assigned versus participant-set goals on skill acquisition and retention of a selected shooting task. J Teach Phys Ed. 1992;11:220-34.

23. Chiviacowsky S, Kaefer A, Medeiros FL, Pereira FM. Aprendizagem motora em crianças: “feedback” após boas tentativas melhora a aprendizagem? Rev Bras Educ Fís Esporte. 2007;21:157-65.

Rev Bras Educ Fís Esporte, (São Paulo) 2014 Out-Dez; 28(4):651-6o • 659 
24. Wrisberg CA, Pein RL. Note on learners' control of the frequency of model presentation during skill acquisition. Percept Mot Skills. 2002;94:792-4.

25. Chiviacowsky S, Wulf G, Lewthwaite R. Self-controlled learning: the importance of protecting perceptions of competence. Front Psychol. 2012;3:458.

\section{Agradecimentos}

A presente pesquisa foi amparada por uma bolsa CAPES para o primeiro autor.

ENDEREÇO

Flavio Henrique Bastos Escola de Educação Física e Esporte - USP Av. Prof. Mello Moraes, 65 05508-030 - São Paulo - SP - BRASIL e-mail: bastosfh@usp.br
Recebido para publicação: 30/01/2014

Revisado: 03/04/2014

Aceito: 18/08/2014 